\title{
Selection Response for the Expression of Quantitative Traits in the Mulberry Silkworm Bombyx mori L.
}

\author{
Shivkumar $^{1 *}$, M.N. Ramya ${ }^{2}$, E. Talebi ${ }^{3}$ and G. Subramanya ${ }^{2}$ \\ ${ }^{1}$ Central Sericultural Research and Training Institute, Central Silk Board, Pampore-192121, \\ Jammu and Kashmir, India \\ ${ }^{2}$ Department of Sericulture, Manasagangothri, University of Mysore, Mysuru-570006, \\ Karnataka, India \\ ${ }^{3}$ Darab branch, Islamic Azad Agriculture University, Darab, Fars, Iran \\ *Corresponding author
}

\begin{abstract}
A B S T R A C T
Selections for quantitative traits are paramount importance to achieve the desired breeding objectives in breed evolving programmes through hybridization. In the light of the above, an experiment was conducted utilizing six different aboriginal silkworm races namely, $\mathrm{C}_{108}, \mathrm{NB}_{4} \mathrm{D}_{2}$, Pure Mysore (PM), Nistari, zebra and knobbed belonging to two voltinistic

\begin{tabular}{|l|}
\hline Ke y w or d s \\
Bombyx mori, \\
Quantitative traits, \\
Selection response, \\
Cellular \& mass \\
selection \\
\hline Article Info \\
\hline Accepted: \\
04 October 2018 \\
Available Online: \\
10 November 2018 \\
\hline \hline
\end{tabular}
groups by rearing them under standard laboratory conditions for six different generations during three seasons of the year. At the parental generations (denoted as $\mathrm{P}_{1}$ ), the harvested cocoons were grouped into two batches wherein, one batch of cocoons were exposed to mass selection and another batch of cocoons were exposed to individual selection by recording data for four important traits namely, cocoon weight, shell weight, filament length and pupation rate. The pooled data was statistically analyzed for analysis of variance (ANOVA) and the results have clearly demonstrated that, the individual (Cellular) selection has resulted in higher increase in the three economic traits $v i z$, cocoon weight, shell weight and filament length significant $(\mathrm{P}<0.05)$, wherein mass selection resulted in significant $(\mathrm{P}<0.05)$ improvement in pupation rate across six generations. The data also revealed that the selection response is high (>96\%) during pre-monsoon season in multivoltine races than the bivoltine races. The obtained data will determine to understand the suitability of selection response for using in genetics and breeding programmes for the production of high yieldable and superior silk content silkworm breeds/hybrids. It is concluded with responses to selection hereby importance of two selection method is discussed in the light of silkworm selection and hybridization programmes.
\end{abstract}

\section{Introduction}

Silkworm, Bombyx mori is an important model organism for various fields such as, scientific, medical and agriculture, etc.,
Silkworm is characterized by four distinct developmental stages namely, egg, larva, pupa and adult. The developmental period from larva to adult in silkworm involves the morphological entrainment and degree of 
rethymic changes at onset of metamorphosis. Sericulture in India, besides providing occupation to about six million people, provides impetus to chief cottage industry. Today, India stands next only to China in global raw silk production. The history of sericulture clearly indicates that silkworm culture was practiced in the Chinese provinces during 2500 BC (Kuhn, 1988). Sericulture began forty five centuries ago in north-eastern part of China along the banks of Hwang Ho River (Hirobe, 1968). In the Indian subcontinent, the concept of production of bivoltine silk is realized during 1970's and four bivoltine races namely NB7, NB18, KA and $\mathrm{NB}_{4} \mathrm{D}_{2}$ become popular at field level.

During 1980's to 2000 several research institutes have made revolutionary work and evolved new productive (CSR series), resistant and season specific breeds/races (bivoltine and multivoltine races) through various concept of conventional breeding and cross breeding approaches in India. The majority of raw silk production from the multivoltine oriented, which is qualitatively inferior in respect to the yield. It is very pertinent to produce more number of productive breeds of bivoltine and cross breeds, which are capable of producing higher quality silk and meet international grade. Henceforth, success of breeding programmes is depends on selection methods and parental materials. As per the selection is considered, there are various avenues of selection like, through biochemical, molecular, tissue level, cell level, morphological, physiological, genetical selection (cellular, mass, family, etc), etc. Further, improvement for traits is paramount step in any breeding programme, which are directly connected to the success in sericulture industry depends on few factors like trait, breed, seed and feed. However, reproductive traits are considered a vital to the egg producers, while cocoon producers are interested in improved production potential, cocoon shell percentage and disease resistant (Singh et al., 1998). On the other hand, as suggested by Seidavi et al., (2008) that, the selection is one of the best breeding approaches in silkworm breeding. Moreover, individual selection in parent lines can improve their offspring performance. But, this improvement is effected to environmental factors specially season (Seidavi, 2010) and concluded his finding with responses to selection for important resistance traits in silkworm pure lines at spring is higher than autumn significantly.

Further, correlation between pedigree and mass selection and traits silkworm (Singh et al., 2011) Suresh Kumar et al., (2013) suggested that, selection is important for breeding materials for development of hybrids suitable to West Bengal. Few researches were done on response to selection in three commercial pure lines with oval cocoon in silkworm Bombyx mori (Hajian et al., 2011).

However, based on the review of literature survey, selection response method is paramount importance in silkworm breeding approach, which is also conducted for several improvement aspects in other insects, animals, poultry, etc, like, swine breeding (Chen et al., 2007), response to selection with an optimum selection and multilevel (Bijma and Muir, 2006, Van Vleck et al., 2007 and Bijma et al., 2007a), bases for individual versus family selection systems (Lush, 1947), theory of limits in artificial selection (Robertson, 1960), influence of selection and mating systems on larval weight in Tribolium (Wilson et al., 1968) genetic gain by selection in annual egg production of the Fowl (Kinney et al., 1970 $\&)$, etc. Generally, when two selected races/strains were crossed in next generation hybrids of selected races become more productive/resistant than compare to the crosses made of unselected strains/races. Henceforth, it is understood through available 
literature in relation to selection methods are play a vital role for deciding the ultimate outcome/production/resistant races under several generations. Thereby, it is in this context, the present research programme is framed/proposed relevant to the response to the selection of cellular and mass rearing during three seasons/six generations for four quantitative traits of the silkworm Bombyx mori.

\section{Materials and Methods}

The present investigation has been carried out on six silkworm commercial pure races/strains, which were drawn from the Germplasm Bank of Department of Sericulture, University of Mysore, Mysuru. The selected races/mutants belonging to two voltinistic group's eggs were incubated with $25 \pm 10^{\circ} \mathrm{c}$ and relative humidity of $80 \pm 5 \%$ and simultaneously black boxing was followed on $8^{\text {th }}$ day to achieve uniformity in hatching.

The standard rearing was conducted during three season viz., pre-monsoon, monsoon and post-monsoon and larvae hatched from each layings were reared separately under uniform laboratory conditions as described by Yokoyama, (1963) and Krishnaswami, (1978).

The rearing was conducted under standard laboratory conditions for six different generations during three seasons of the year and evaluated for four important quantitative traits to understand the suitability of selection response for using in genetics and breeding programmes for the production of high yielding and superior silk content silkworm breeds/hybrids.

At the parental generations, the harvested cocoons were grouped into two batches wherein, one batch of cocoons were exposed to mass selection and another batch of cocoons were exposed to individual selection by recording data for four important traits namely, cocoon weight, shell weight, filament length and pupation rate., The following formulae were adopted for analysis of selection response by manually to determine the improvement/response for selection during six generation.

Improvement of selection response at $\mathrm{F} 1$ over P1 generation

Value of F1 - value of P1 = improvement at F1 over P1 generation

Percentage improvement of selection response at F1 over P1 generation

[Value of improvement at F1 over P1 / P1 value] $\mathrm{x} 100=\%$ improvement at $\mathrm{F} 1$ over P1 generation

Improvement of selection response at $\mathrm{F} 2$ over F1 generation

Value of F2 - value of F1 = improvement at $\mathrm{F} 2$ over $\mathrm{F} 1$ generation

Percentage improvement of selection response at F2 over F1 generation

[Value of improvement at F2 over F1 / F1 value] $\mathrm{x} 100=\%$ improvement at $\mathrm{F} 2$ over $\mathrm{F} 1$ generation

Improvement of selection response at $\mathrm{F} 3$ over $\mathrm{P} 1$ generation

Value of F3 - value of P1 = improvement at $\mathrm{F} 3$ over P1 generation

Percentage improvement of selection response at F3 over P1 generation

[Value of improvement at F3 over P1 / P1 value] $\mathrm{x} 100=\%$ improvement at $\mathrm{F} 3$ over P1generation 
Improvement of selection response at F3 over F2 generation

Value of F3 - value of F2 = improvement at F3 over F2 generation

Percentage improvement of selection response at $\mathrm{F} 3$ over $\mathrm{F} 2$ generation

[Value of improvement at F3 over F2 / F2 value] $\mathrm{x} 100=\%$ improvement at $\mathrm{F} 3$ over $\mathrm{F} 2$ generation

Improvement of selection response at F4 over P1 generation

Value of F4 - value of P1 = improvement at F4 over P1 generation

Percentage improvement of selection response at F4 over P1 generation

[Value of improvement at F4 over P1 / P1 value] $\mathrm{x} 100=\%$ improvement at $\mathrm{F} 4$ over P1 generation

Improvement of selection response at F4 over F3 generation

Value of F4 - value of F3 = improvement at F4 over F3 generation

Percentage improvement of selection response at F4 over F3 generation

[Value of improvement at F4 over F3 / F3 value] $x 100=\%$ improvement at $\mathrm{F} 4$ over $\mathrm{F} 3$ generation

Improvement of selection response at F5 over P1 generation

Value of F5 - value of P1 = improvement at F5 over P1 generation

Percentage improvement of selection response at F5 over P1 generation
[Value of improvement at F5 over P1 / P1 value] $\mathrm{x} 100=\%$ improvement at F5 over P1 generation

Improvement of selection response at F5 over F4 generation

Value of F5 - value of F4 = improvement at F5 over F4 generation

Percentage improvement of selection response at F5 over F4 generation

[Value of improvement at F5 over F4 / F4 value] $\mathrm{x} 100=\%$ improvement at $\mathrm{F} 5$ over $\mathrm{F} 4$ generation

Improvement of selection response at F6 over P1 generation

Value of F6 - value of P1 = improvement at F6 over P1 generation

Percentage improvement of selection response at F6 over P1 generation

[Value of improvement at F6 over P1 / $\mathrm{P} 1$ value] $\mathrm{x} 100=\%$ improvement at F6 over P1 generation

Improvement of selection response at F6 over F5 generation

Value of F6 - value of F5 = improvement at F6 over F5 generation

Percentage improvement of selection response at F6 over F5 generation

[Value of improvement at F6 over F5 / F5value] x $100=\%$ improvement at $\mathrm{F} 6$ over F5 generation

The obtained data pertaining to four quantitative traits of six generation from the experiment thereby were statistically analyzed through OPISTAT statistical package. 


\section{Results and Discussion}

The success of selection is monitored by the degree to which the desired trait is transmitted to the succeeding generation and nature of selection is to be given due to consideration at appropriate developmental stages for pursuing selection in desired direction, while improving or evolving high productive breeds or hybrids of the silkworm Bombyx mori. The results of the selection response with the improvement \& percent improvement across six generation studies utilizing two each of bivoltine, multivoltine and mutants of the silkworm Bombyx mori depicted in the Table 1. It shows rearing performance and improvement across six generation through cellular rearing method for four quantitative traits of the silkworm (Figures 1-4). The results in regard to cocoon weight of $\mathrm{C} 108$ race recorded of $1.88 \mathrm{~g}$ at $\mathrm{P} 1$ level followed by $\mathrm{NB}_{4} \mathrm{D}_{2}$ (1.75g), PM (1.03g), Nistari $(0.88 \mathrm{~g})$, zebra $(1.60 \mathrm{~g})$ and knobbed (1.510g).

Whereas an increase of $1.91 \mathrm{~g}$ at $\mathrm{F} 1$ generation in $\mathrm{C}_{108}$ and showed improvement of $0.03 \mathrm{~g}$ over the P1. Further, all other races also showed increase of cocoon weight at F1 compare to the P1 level (Fig. 1). Moreover, there was a slight increase in F2 (1.94g), F3 generation (1.96g), F4 (1.99g), F5 (2.01) and F6 (2.11g), which showing improvement of 0.06g (F1 over P1), 0.03g (F2 over F1), 0.08g (F3 over P1), $0.02 \mathrm{~g}$ (F3 over F2), $0.11 \mathrm{~g}$ (F4 over P1), 0.03g (F4 over F3), 0.13g (F5 over P1), 0.02g (F5 over F4), 0.23g (F6 over P1), $0.1 \mathrm{~g}$ (F6 over F5) with the percent improvement of $12.23 \%$ at F6 over P1and $4.97 \%$ at $\mathrm{F} 6$ over $\mathrm{F} 5$ respectively.

Further, an average was made across the six generation and recorded of $1.98 \mathrm{~g}$ cocoon weight in $\mathrm{C}_{108}$ race. More interestingly, for the traits like shell weight (Fig. 2) and filament length (Fig. 3) showed the same trend of increase $\mathrm{g} / \mathrm{mtr}$ from $\mathrm{P} 1$ level of $0.315 \mathrm{~g}$ shell weight \& 900mtr filament length to the F6 generation of $0.401 \mathrm{~g}$ shell weight \& $1201 \mathrm{mtr}$ length of filament respectively. But, the trend of increasing $\mathrm{g} / \mathrm{mtr}$ was not evident in case of pupation rate (Fig. 4) instead decreasing of pupation rate consistently in every generation from P1 (93.11\%) level to $\mathrm{F} 6$ generation $(91.05 \%)$ in $\mathrm{C}_{108}$ race. Further, the same trend of increasing $\mathrm{g} / \mathrm{mtr}$ traits like cocoon weight, shell weight and filament length was observed and decreasing phenomenon was noticed in case of pupation rate in $\mathrm{NB}_{4} \mathrm{D}_{2}$ race, Pure Mysore, Nistari, zebra \& knobbed races/mutants through cellular selection rearing method (Table 1). Few investigators have worked for selection for region and season specific breeds/hybrids are $\mathrm{He}$ and Oshiki (1984), Nirmal Kumar (1995), Sudhakar Rao (2003), Suresh Kumar et al., (2013), etc.

Further, the data in regards to Table 2 showing rearing performance and improvement over the generations through mass rearing method for the four quantitative traits (cocoon weight, shell weight \& filament length) of the silkworm Bombyx mori. The results for trait cocoon weight recorded of $1.88 \mathrm{~g}$ at P1 level, $1.95 \mathrm{~g}$ at $\mathrm{F} 1$ generation \& $1.98 \mathrm{~g}$ during $\mathrm{F} 2$ generation recorded accordingly, but during F3 generation, it was noticed $1.88 \mathrm{~g}$ cocoon weight occurring of inbreeding depression at F3 \& again it was increased for $2.00 \mathrm{~g}$ at F4 generation then again showing inbreeding depression during F5 of 1.90g) \& F6 of $1.82 \mathrm{~g}$ respectively. However, interestingly this trend of heterosis at F1 (1.95g), F2 (1.98g) \& F4 $(2.00 \mathrm{~g})$ occurred at the same time, the trend of inbreeding depression was evident at F3 generation (1.88g), F5 (1.90g) \& F6 (1.82g) compare to earlier generations in $\mathrm{C}_{108}$ race and this was relevant with the results of Dobzhansky, (1950), who has worked on origin of heterosis through natural selection in populations Drosophila pseudobscura. 


\section{Int.J.Curr.Microbiol.App.Sci (2018) 7(11): 3456-3474}

Table.1 Rearing performance \& improvement over the generations by cellular rearing for four quantitative traits of the silkworm

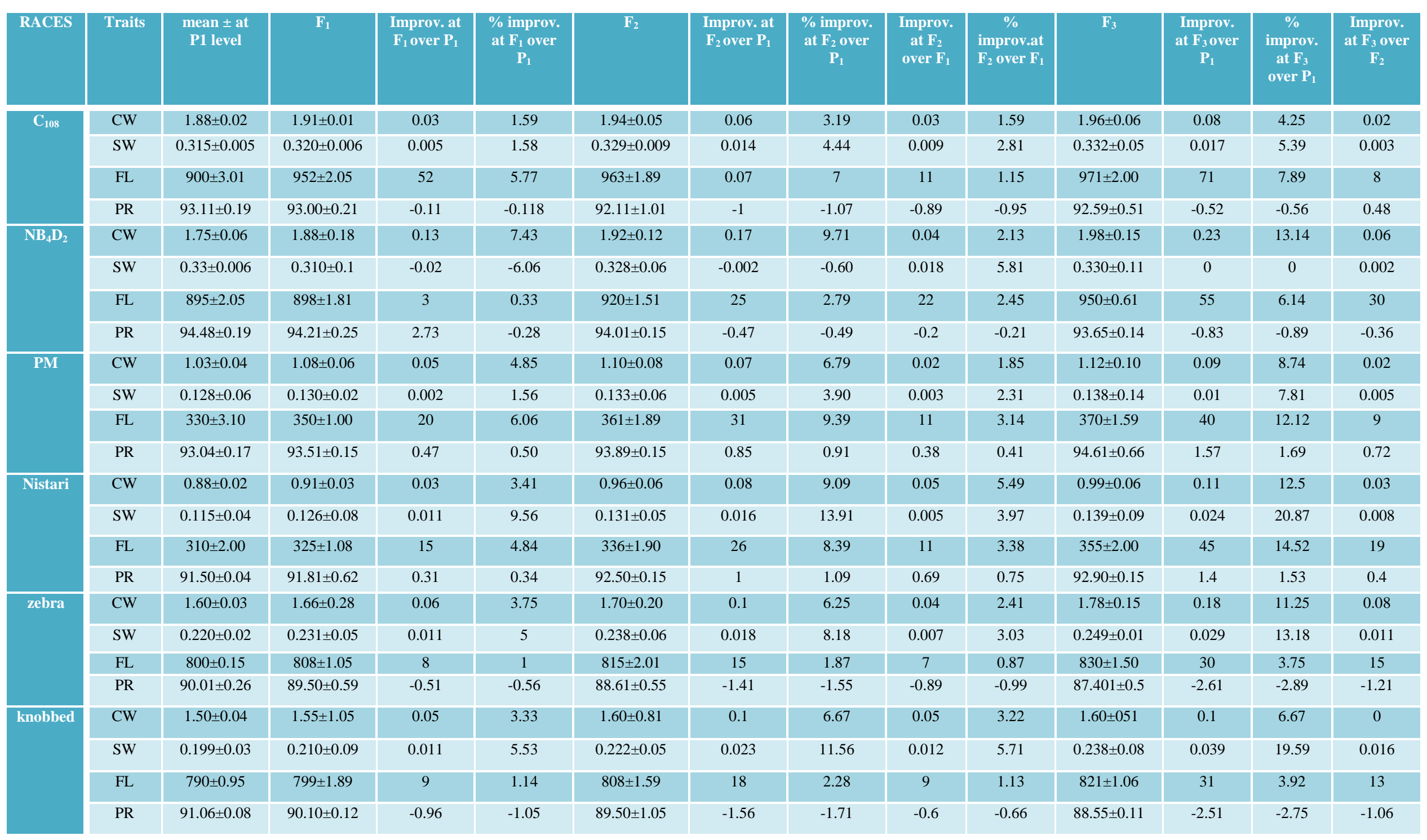


Int.J.Curr.Microbiol.App.Sci (2018) 7(11): 3456-3474

\begin{tabular}{|c|c|c|c|c|c|c|c|c|c|c|c|c|c|c|c|c|}
\hline $\begin{array}{c}\% \text { improv. } \\
\text { at } F_{3} \text { over } \\
F_{2}\end{array}$ & $\mathbf{F}_{4}$ & $\begin{array}{l}\text { Impro } \\
\text { v. at } F_{4} \\
\text { over } P_{1}\end{array}$ & $\begin{array}{c}\% \text { improv. } \\
\text { at } \mathbf{F}_{4} \text { over } \\
\mathbf{P}_{1}\end{array}$ & $\begin{array}{l}\text { Improv. at } \\
F_{4} \text { over } F_{3}\end{array}$ & $\begin{array}{c}\% \text { improv. } \\
\text { at } F_{4} \\
\text { over } F_{3}\end{array}$ & $\mathbf{F}_{5}$ & $\begin{array}{l}\text { Impro } \\
\text { v. at } F_{5} \\
\text { over } P_{1}\end{array}$ & $\begin{array}{l}\text { Impro } \\
\text { v. at } F_{5} \\
\text { over } F_{4}\end{array}$ & $\begin{array}{c}\% \\
\text { improv. } \\
\text { at } F_{5} \\
\text { over } P_{1}\end{array}$ & $\begin{array}{c}\% \\
\text { improv. } \\
\text { at } F_{5} \\
\text { over } F_{4}\end{array}$ & $F_{6}$ & $\begin{array}{c}\text { Improv. } \\
\text { at } \mathrm{F}_{6} \text { over } \\
\mathbf{P}_{1}\end{array}$ & $\begin{array}{c}\% \\
\text { improv } \\
\text {. at } \mathrm{F}_{6} \\
\text { over } \mathrm{P}_{1}\end{array}$ & $\begin{array}{c}\text { Improv. } \\
\text { at } F_{6} \\
\text { over } F_{5}\end{array}$ & $\begin{array}{c}\% \\
\text { improv } \\
\text {-at } F_{6} \\
\text { over } F_{5}\end{array}$ & $\begin{array}{c}\text { Average of } \\
6 \\
\text { generation }\end{array}$ \\
\hline 1.03 & $1.99 \pm 0.08$ & 0.11 & 5.85 & 0.03 & 1.53 & $2.01 \pm 0.09$ & 0.13 & 0.02 & 1.00 & 6.91 & $2.11 \pm 0.08$ & 0.23 & 12.23 & 0.1 & 4.97 & 1.986 \\
\hline 0.91 & $0.349 \pm 0.04$ & 0.034 & 10.79 & 0.017 & 5.12 & $0.376 \pm 0.08$ & 0.061 & 0.027 & 7.74 & 19.36 & $0.401 \pm 0.02$ & 0.086 & 27.30 & 6.025 & 6.65 & 0.351 \\
\hline 0.83 & $999 \pm 2.12$ & 99 & 11 & -71 & -7.31 & $1105 \pm 2.01$ & 205 & 106 & 10.61 & 22.78 & $1201 \pm 2.10$ & 301 & 33.44 & 95 & 8.68 & 1032 \\
\hline 0.52 & $92.81 \pm 1.51$ & -0.3 & -0.32 & 0.22 & 0.24 & $91.61 \pm 1.15$ & -1.5 & -1.2 & -1.29 & -1.61 & $91.05 \pm 0.51$ & -2.06 & -2.21 & -0.56 & -0.61 & 92.19 \\
\hline 3.12 & $2.04 \pm 0.26$ & 0.29 & 16.56 & 0.06 & 3.03 & $2.09 \pm 0.12$ & 0.34 & 0.05 & 2.45 & 19.43 & $2.18 \pm 0.08$ & 0.46 & 24.57 & 0.09 & 4.31 & 2.015 \\
\hline 0.609 & $0.340 \pm 0.06$ & 0.01 & 3.03 & 0.01 & 3.03 & $0.360 \pm 0.06$ & 0.03 & 0.02 & 5.88 & 9.09 & $0.400 \pm 0.09$ & 0.07 & 21.21 & 0.04 & 11.11 & 0.344 \\
\hline 3.26 & $996 \pm 2.89$ & 101 & 11.28 & 46 & 4.84 & $1191 \pm 2.01$ & 296 & 195 & 19.58 & 33.07 & $1200 \pm 1.89$ & 305 & 34.08 & 9 & 0.75 & 1025 \\
\hline-0.38 & $93.45 \pm 0.05$ & -1.03 & -1.09 & -0.2 & -0.21 & $93.25 \pm 0.15$ & -1.23 & -0.2 & -0.21 & -1.30 & $93.10 \pm 0.06$ & -1.38 & -1.46 & -0.15 & -0.16 & 93.61 \\
\hline 1.82 & $1.13 \pm 0.20$ & 0.1 & 9.71 & 0.01 & 0.89 & $1.15 \pm 1.00$ & 0.12 & 0.02 & 1.77 & 11.65 & $1.25 \pm 0.25$ & 0.22 & 21.36 & 0.1 & 8.69 & 1.138 \\
\hline 3.76 & $0.140 \pm 0.01$ & 0.012 & 9.37 & 0.002 & 1.45 & $0.146 \pm 0.06$ & 0.018 & 0.006 & 4.28 & 14.06 & $0.150 \pm 0.07$ & 0.022 & 17.19 & 0.004 & 2.74 & 0.139 \\
\hline 3.49 & $379 \pm 1.61$ & 49 & 14.85 & 9 & 2.43 & $385 \pm 2.65$ & 55 & 6 & 1.58 & 16.67 & $406 \pm 3.05$ & 76 & 23.03 & 21 & 5.45 & 375 \\
\hline 0.77 & $94.90 \pm 0.04$ & 1.86 & 1.99 & 0.29 & 0.31 & $95.80 \pm 0.66$ & 2.76 & 0.9 & 0.95 & 2.96 & $95.98 \pm 0.69$ & 2.94 & 3.16 & 0.18 & 0.19 & 94.78 \\
\hline 3.12 & $1.05 \pm 0.02$ & 0.17 & 19.32 & 0.06 & 6.06 & $1.11 \pm 0.06$ & 0.23 & 0.006 & 5.71 & 26.14 & $1.20 \pm 0.25$ & 0.32 & 36.36 & 0.09 & 8.11 & 1.036 \\
\hline 6.11 & $0.142 \pm 0.05$ & 0.027 & 23.48 & 0.003 & 2.16 & $0.144 \pm 0.05$ & 0.029 & 0.002 & 1.41 & 25.22 & $0.148 \pm 0.08$ & 0.033 & 28.69 & 0.004 & 2.78 & 0.138 \\
\hline 5.56 & $370 \pm 1.40$ & 60 & 19.35 & 15 & 4.22 & $378 \pm 1.05$ & 68 & 8 & 2.16 & 21.93 & $388 \pm 2.06$ & 78 & 25.16 & 10 & 2.64 & 358 \\
\hline 0.42 & $93.40 \pm 0.08$ & 1.9 & 2.08 & 0.5 & 0.54 & $93.89 \pm 0.59$ & 2.39 & 0.49 & 0.52 & 2.61 & $94.49 \pm 0.15$ & 2.99 & 3.27 & 0.6 & 0.64 & 93.16 \\
\hline 4.70 & $1.85 \pm 1.50$ & 0.25 & 15.62 & 0.07 & 3.93 & $1.86 \pm 0.52$ & 0.26 & 0.01 & 0.54 & 16.25 & $1.84 \pm 0.70 .3$ & 0.24 & 15 & -0.02 & -0.07 & 1.782 \\
\hline 4.62 & $0.258 \pm 0.10$ & 0.038 & 17.27 & 0.009 & 3.61 & $0.278 \pm 0.09$ & 0.058 & 0.02 & 7.75 & 26.36 & $0.310 \pm 0.07$ & 0.09 & 40.90 & 0.032 & 11.51 & 0.261 \\
\hline 1.84 & $841 \pm 0.60$ & 41 & 5.12 & 11 & 1.32 & $862 \pm 1.60$ & 62 & 21 & 2.49 & 7.75 & $860 \pm 1.55$ & 60 & 7.5 & -2 & -0.23 & 836 \\
\hline-1.36 & $87.01 \pm 0.55$ & -3.01 & -3.34 & -0.4 & -0.46 & $86.59 \pm 0.69$ & -3.42 & -0.41 & -0.49 & -3.79 & $86.00 \pm 0.05$ & -4.01 & -4.45 & -0.59 & -0.68 & 87.52 \\
\hline 0 & $1.68 \pm 0.50$ & 0.18 & 12 & 0.08 & 5 & $1.69 \pm 0.89$ & 0.19 & 0.01 & 0.59 & 12.67 & $1.71 \pm 0.15$ & 0.21 & 14 & 0.02 & 1.18 & 1.638 \\
\hline 7.21 & $0.248 \pm 0.15$ & 0.049 & 24.62 & 0.01 & 4.20 & $0.260 \pm 0.06$ & 0.061 & 0.012 & 4.84 & 30.65 & $0.291 \pm 0.15$ & 0.092 & 46.23 & 0.031 & 11.92 & 0.245 \\
\hline 1.61 & $829 \pm 1.049$ & 38 & 4.94 & 8 & 0.97 & $836 \pm 1.44$ & 46 & 7 & 0.84 & 5.82 & $851 \pm 1.45$ & 61 & 7.72 & 15 & 1.79 & 824 \\
\hline-1.06 & $87.40 \pm 0.52$ & -3.66 & -4.02 & -1.15 & -1.31 & $86.50 \pm 0.06$ & -4.56 & -0.9 & -1.03 & -5.01 & $86.00 \pm 0.50$ & -5.06 & -5.56 & -0.5 & -0.58 & 88.17 \\
\hline
\end{tabular}




\section{Int.J.Curr.Microbiol.App.Sci (2018) 7(11): 3456-3474}

Table.2 Rearing performance \& improvement over the generations by mass rearing for four quantitative traits of the silkworm

\begin{tabular}{|c|c|c|c|c|c|c|c|c|c|c|c|c|c|c|}
\hline RACES & Traits & $\begin{array}{c}\text { mean } \pm \text { at } \mathrm{p} 1 \\
\text { level }\end{array}$ & $F_{1}$ & $\begin{array}{c}\text { Impr. at } F_{1} \\
\text { over } P_{1}\end{array}$ & $\begin{array}{c}\% \text { impr. at } \mathbf{F}_{1} \\
\text { over } \mathbf{P}_{1}\end{array}$ & $\mathbf{F}_{2}$ & $\begin{array}{c}\text { Impr. at } F_{2} \\
\text { over } P_{1}\end{array}$ & $\begin{array}{l}\% \text { impr. at } \\
F_{2} \text { over } P_{1}\end{array}$ & $\begin{array}{c}\text { Impr. at } F_{2} \\
\text { over } F_{1}\end{array}$ & $\begin{array}{l}\% \text { impr. at } \\
\mathrm{F}_{2} \text { over } \mathrm{F}_{1}\end{array}$ & $F_{3}$ & $\begin{array}{l}\text { Impr. } \\
\text { at } F_{3} \\
\text { over } P_{1}\end{array}$ & $\begin{array}{c}\% \text { impr. } \\
\text { at } \mathbf{F}_{3} \\
\text { over } \mathbf{P}_{1}\end{array}$ & $\begin{array}{c}\text { Impr. at } \\
F_{3} \text { over } \\
F_{2}\end{array}$ \\
\hline \multirow[t]{4}{*}{$\mathbf{C}_{108}$} & $\mathrm{CW}$ & $1.88 \pm 0.02$ & $1.95 \pm 0.15$ & 0.07 & 3.723 & $1.98 \pm 0.11$ & 0.10 & 5.319 & -0.03 & -1.538 & $1.88 \pm 0.16$ & 00 & 00 & -0.1 \\
\hline & SW & $0.315 \pm 0.005$ & $0.335 \pm 0.51$ & 0.023 & 7.301 & $0.330 \pm 0.50$ & 0.015 & 4.761 & 0.005 & 1.492 & $0.350 \pm 0.11$ & -0.035 & -11.111 & 0.02 \\
\hline & FL & $900 \pm 3.01$ & $1000 \pm 1.25$ & 100 & 11.111 & $995 \pm 1.89$ & 95 & 10.555 & -5 & -0.5 & $1001 \pm 0.91$ & -100 & -11.111 & 6 \\
\hline & PR & $93.11 \pm 0.19$ & $94.09 \pm 1.66$ & 1.78 & 1.911 & $94.85 \pm 0.55$ & 1.74 & 1.868 & 0.75 & 0.807 & $94.88 \pm 0.65$ & 0.77 & 0.827 & -0.97 \\
\hline \multirow[t]{4}{*}{$\mathrm{NB}_{4} \mathbf{D}_{2}$} & $\mathrm{CW}$ & $1.75 \pm 0.06$ & $1.91 \pm 0.15$ & 0.161 & 9.142 & $1.87 \pm 0.55$ & 0.12 & 6.857 & 0.04 & 2.094 & $1.95 \pm 0.15$ & 0.20 & 11.428 & -0.08 \\
\hline & SW & $0.33 \pm 0.006$ & $0.326 \pm 0.11$ & -0.004 & -1.212 & $0.320 \pm 0.61$ & -0.01 & -3.030 & 0.006 & 1.840 & $0.330 \pm 0.55$ & 00 & 00 & -0.01 \\
\hline & FL & $895 \pm 2.05$ & $980 \pm 3.55$ & 85 & 9.497 & $990 \pm 1.55$ & 95 & 10.614 & 10 & 1.020 & $999 \pm 1.55$ & 104 & 11.620 & -9 \\
\hline & PR & $94.38 \pm 0.19$ & $94.45 \pm 0.55$ & 0.07 & 0.074 & $94.50 \pm 0.15$ & 0.12 & 0.127 & -0.05 & -0.053 & $94.80 \pm 0.15$ & 0.58 & 0.720 & -0.7 \\
\hline \multirow[t]{4}{*}{ PM } & $\mathrm{CW}$ & $1.03 \pm 0.04$ & $1.10 \pm 0.06$ & 0.07 & 6.796 & $1.12 \pm 0.05$ & 0.09 & 8.737 & 0.02 & 1.818 & $1.09 \pm 0.16$ & 0.06 & 5.825 & -0.03 \\
\hline & SW & $0.128 \pm 0.06$ & $0.138 \pm 0.01$ & 0.01 & 7.812 & $0.140 \pm 0.15$ & 0.012 & 9.375 & 0.048 & 25.532 & $0.131 \pm 0.14$ & 0.003 & 2.343 & -0.009 \\
\hline & FL & $330 \pm 3.10$ & $360 \pm 1.55$ & 30 & 9.090 & $368 \pm 2.61$ & 38 & 11.515 & -8 & -2.222 & $361 \pm 1.33$ & 31 & 9.394 & -7 \\
\hline & PR & $93.04 \pm 0.17$ & $93.55 \pm 0.15$ & .51 & 0.548 & $94.23 \pm 1.55$ & 1.19 & 1.279 & -.68 & -.727 & $94.60 \pm 0.05$ & -0.56 & -0.602 & -0.63 \\
\hline \multirow[t]{4}{*}{ Nistari } & $\mathrm{CW}$ & $0.88 \pm 0.02$ & $0.99 \pm 0.16$ & 0.11 & 12.5 & $1.05 \pm 0.10$ & 0.17 & 19.318 & 0.06 & 6.061 & $0.95 \pm 0.05$ & 0.07 & 7.954 & -0.1 \\
\hline & SW & $0.115 \pm 0.04$ & $0.126 \pm 0.01$ & 0.01 & 9.565 & $0.131 \pm 0.05$ & 0.016 & 13.913 & 0.005 & 3.969 & $0.121 \pm 0.15$ & -0.006 & -5.219 & -0.01 \\
\hline & FL & $310 \pm 2.00$ & $340 \pm 1.55$ & 30 & 9.677 & $365 \pm 2.15$ & 55 & 17.742 & 25 & 7.353 & $341 \pm 2.17$ & 31 & 10 & 24 \\
\hline & PR & $91.50 \pm 0.04$ & $92.00 \pm 1.11$ & 0.5 & 0.546 & $93.25 \pm 0.21$ & 1.75 & 1.912 & -1.25 & -1.358 & $93.31 \pm 0.17$ & -0.29 & -0.317 & 2.04 \\
\hline \multirow[t]{4}{*}{ zebra } & $\mathrm{CW}$ & $1.60 \pm 0.03$ & $1.74 \pm 0.15$ & 0.14 & 8.750 & $1.65 \pm 0.16$ & 0.05 & 3.125 & 0.09 & 5.172 & $1.71 \pm 0.18$ & 0.11 & 6.875 & 0.06 \\
\hline & SW & $0.220 \pm 0.02$ & $0.290 \pm 0.16$ & 0.07 & 31.818 & $0.290 \pm 0.55$ & 0.07 & 31.818 & 00 & 00 & $0.298 \pm 0.15$ & -0.078 & -35.45 & -0.008 \\
\hline & FL & $800 \pm 0.15$ & $855 \pm 1.11$ & 55 & 6.875 & $855 \pm 1.00$ & 55 & 6.875 & 00 & 00 & $860 \pm 2.15$ & 60 & 7.5 & 5 \\
\hline & PR & $90.01 \pm 0.26$ & $90.55 \pm 0.11$ & 0.65 & 0.722 & $91.56 \pm 0.11$ & 1.55 & 1.722 & -1.01 & -1.115 & $91.50 \pm 0.12$ & -0.51 & -0.566 & -2.06 \\
\hline \multirow[t]{4}{*}{ knobbed } & $\mathrm{CW}$ & $1.50 \pm 0.04$ & $1.61 \pm 0.15$ & 0.11 & 7.333 & $1.68 \pm 0.11$ & -0.18 & -12.00 & 0.07 & 4.348 & $1.58 \pm 0.24$ & 0.08 & 5.333 & -0.1 \\
\hline & SW & $0.199 \pm 0.03$ & $0.210 \pm 0.22$ & 0.011 & 5.527 & $0.230 \pm 0.55$ & 0.031 & 15.577 & -0.02 & -9.524 & $0.221 \pm 0.15$ & 0.022 & 11.055 & -0.009 \\
\hline & FL & $790 \pm 0.95$ & $799 \pm 2.00$ & 9 & 1.139 & $790 \pm 1.55$ & 10 & 1.266 & -1 & -0.125 & $798 \pm 0.61$ & 8 & 1.013 & 2 \\
\hline & PR & $90.05 \pm 0.08$ & $91.59 \pm 0.11$ & 0.53 & 0.582 & $92.06 \pm 0.35$ & 1.00 & 1.098 & 0.46 & 0.502 & $92.65 \pm 0.25$ & 0.41 & 0.450 & -1.41 \\
\hline
\end{tabular}


Int.J.Curr.Microbiol.App.Sci (2018) 7(11): 3456-3474

\begin{tabular}{|c|c|c|c|c|c|c|c|c|c|c|c|c|c|c|c|c|}
\hline $\begin{array}{l}\% \text { impr. at } \\
F_{3} \text { over } F_{2}\end{array}$ & $\mathbf{F}_{4}$ & $\begin{array}{c}\text { Improv } \\
\text { at } F_{4} \\
\text { over } P_{1}\end{array}$ & $\begin{array}{c}\% \\
\text { improv. } \\
\text { at } \mathbf{F}_{4} \text { over } \\
\mathbf{P}_{1}\end{array}$ & $\begin{array}{c}\text { Improv. } \\
\text { at } \mathrm{F}_{4} \\
\text { over } \mathrm{F}_{3}\end{array}$ & $\begin{array}{c}\% \\
\text { improv. } \\
\text { at } F_{4} \\
\text { over } F_{3}\end{array}$ & $\mathbf{F}_{5}$ & $\begin{array}{l}\text { Impro } \\
\text { v. at } F_{5} \\
\text { over } P_{1}\end{array}$ & $\begin{array}{l}\text { Impro } \\
\text { v. at } F_{5} \\
\text { over } F_{4}\end{array}$ & $\begin{array}{c}\% \\
\text { impro } \\
\text { v. at } \mathbf{F}_{5} \\
\text { over } \mathbf{F}_{4}\end{array}$ & $\begin{array}{c}\% \\
\text { improv } \\
\text {-at } F_{5} \\
\text { over } P_{1}\end{array}$ & $F_{6}$ & $\begin{array}{c}\text { Improv. } \\
\text { at } \mathrm{F}_{6} \text { over } \\
\mathbf{P}_{1}\end{array}$ & $\begin{array}{c}\% \\
\text { improv. } \\
\text { at } \mathrm{F}_{6} \\
\text { over } \mathrm{P}_{1}\end{array}$ & $\begin{array}{c}\text { Improv. } \\
\text { at } F_{6} \\
\text { over } F_{5}\end{array}$ & $\begin{array}{c}\% \\
\text { improv } \\
\text {. at } \mathrm{F}_{6} \\
\text { over } \mathrm{F}_{5}\end{array}$ & $\begin{array}{c}\text { Averag } \\
\text { e of } 6 \\
\text { genera } \\
\text { tion }\end{array}$ \\
\hline-5.050 & $2.00 \pm 0.17$ & 0.12 & 6.382 & 0.12 & 6.00 & $1.90 \pm 0.61$ & 0.02 & 1.052 & -0.1 & -5.263 & $1.82 \pm 0.15$ & -0.06 & -3.296 & -0.08 & -4.395 & 1.921 \\
\hline 6.060 & $0.390 \pm 0.55$ & 0.075 & 23.809 & 0.04 & 11.428 & $0.352 \pm 0.51$ & 0.037 & 10.511 & -0.038 & -10.795 & $0.338 \pm 0.08$ & 0.023 & 6.804 & -0.014 & -4.142 & 0.349 \\
\hline 0.603 & $1101 \pm 2.15$ & 201 & 22.333 & 100 & 10.010 & $998 \pm 2.15$ & 98 & 9.819 & -103 & -10.320 & $988 \pm 1.51$ & 88 & 8.907 & -10.00 & 1.012 & 10.13 \\
\hline-1.022 & $94.88 \pm 0.15$ & 1.04 & 1.117 & 0.27 & 0.287 & $95.25 \pm 0.22$ & 2.14 & 2.246 & 1.1 & 1.155 & $96.55 \pm 0.15$ & 3.44 & 3.563 & 1.3 & 1.346 & 94.795 \\
\hline-4.278 & $2.05 \pm 0.05$ & 0.3 & 17.142 & 0.1 & 5.128 & $1.90 \pm 1.66$ & 0.15 & 7.895 & -0.15 & -7.895 & $1.80 \pm 0.14$ & 0.05 & 2.777 & -0.1 & -5.55 & 1.913 \\
\hline-3.125 & $0.391 \pm 0.17$ & 0.061 & 18.485 & 0.061 & 18.484 & $0.380 \pm 0.28$ & 0.05 & 13.157 & -0.011 & -2.895 & $0.361 \pm 0.59$ & 0.031 & 8.587 & -0.019 & -5.263 & 0.351 \\
\hline-0.909 & $1100 \pm 0.14$ & 205 & 22.905 & 101 & 10.110 & $998 \pm 1.56$ & 103 & 10.320 & -102 & -10220 & $950 \pm 3.15$ & 55 & 5.789 & -48 & -5.052 & 986 \\
\hline-0.740 & $94.81 \pm 0.14$ & 0.13 & 0.551 & 0.71 & 0.756 & $95.00 \pm 0.17$ & 0.62 & 0.652 & 0.49 & 0.515 & $95.88 \pm 0.61$ & 1.5 & 1.564 & 0.88 & 0.918 & 94.69 \\
\hline-2.678 & $1.15 \pm 0.28$ & 0.120 & 11.650 & 0.06 & 5.504 & $1.10 \pm 0.15$ & 0.07 & 6.363 & -0.05 & -4.545 & $1.09 \pm 0.12$ & 0.06 & 5.504 & -0.01 & -0.917 & 1.442 \\
\hline-6.428 & $0.141 \pm 0.10$ & 0.013 & 10.156 & 0.01 & 7.633 & $0.132 \pm 0.11$ & 0.004 & 3.030 & -0.009 & -6.818 & $0.130 \pm 0.21$ & 0.002 & 1.538 & -0.002 & -1.538 & 0.144 \\
\hline-1.902 & $395 \pm 1.50$ & 65 & 19.696 & 34 & 9.418 & $389 \pm 0.16$ & 59 & 15.167 & -6 & -1.507 & $370 \pm 3.15$ & 40 & 10.811 & -19.00 & -5.135 & 374 \\
\hline-0.668 & $94.60 \pm 0.55$ & 0.96 & 1.032 & 0.4 & 0.427 & $94.75 \pm 1.15$ & 1.52 & 1.607 & 0.55 & 0.582 & $95.00 \pm 0.18$ & 1.96 & 2.063 & 0.45 & 0.473 & 94.155 \\
\hline-9.523 & $1.10 \pm 015$ & 0.22 & 25.00 & 0.15 & 15.789 & $0.96 \pm 0.21$ & 0.08 & 8.333 & -0.14 & -14.583 & $0.90 \pm 0.15$ & 0.02 & 2.222 & -0.06 & -6.666 & 0.993 \\
\hline-7.633 & $0.132 \pm 0.15$ & 0.017 & 14.782 & 0.011 & 9.090 & $0.120 \pm 0.15$ & 0.005 & 4.166 & -0.012 & -10.00 & $0.119 \pm 0.11$ & 0.004 & 3.361 & -0.001 & -0.840 & 0.125 \\
\hline 6.575 & $375 \pm 1.55$ & 65 & 20.967 & 34 & 9.970 & $365 \pm 1.01$ & 55 & 15.068 & -10.0 & -2.739 & $356 \pm 2.30$ & 46 & 12.921 & -9 & -2.528 & 357 \\
\hline 2.187 & $93.65 \pm 0.25$ & 2.15 & 2.349 & 2.44 & 2.675 & $93.75 \pm 0.15$ & 2.05 & 2.191 & -0.1 & -0.107 & $94.05 \pm 0.15$ & 2.55 & 2.711 & 0.5 & 0.531 & 92.95 \\
\hline 3.636 & $1.79 \pm 0.14$ & 0.19 & 11.875 & 0.08 & 4.678 & $1.70 \pm 0.12$ & 0.1 & 5.882 & -0.09 & -5.294 & $1.65 \pm 0.11$ & 0.05 & 3.030 & -0.05 & -3.030 & 1.706 \\
\hline-2.758 & $0.302 \pm 0.15$ & 0.082 & 37.272 & 0.004 & 1.342 & $0.289 \pm 0.16$ & 0.069 & 23.875 & -0.013 & -4.498 & $0.278 \pm 0.66$ & 0.058 & 20.863 & -0.011 & -3.956 & 0.291 \\
\hline 0.585 & $865 \pm 1.11$ & 65 & 0.125 & 5 & 0.581 & $860 \pm 1.17$ & 60 & 6.976 & -5 & -0.581 & $830 \pm 2.15$ & 30 & 3.614 & -30 & -3.614 & 854 \\
\hline-2.249 & $91.40 \pm 1.16$ & 0.39 & 0.433 & 0.9 & 1.005 & $91.50 \pm 1.12$ & 0.49 & 0.542 & 0.10 & 0.110 & $91.55 \pm 0.12$ & 1.54 & 1.682 & 1.05 & 1.147 & 90.69 \\
\hline-5.952 & $1.69 \pm 0.12$ & 0.19 & 12.666 & 0.11 & 6.962 & $1.60 \pm 0.14$ & 0.10 & 6.25 & -0.009 & -0.09 & $1.58 \pm 0.16$ & 0.08 & 5.063 & -0.02 & -1.266 & 1.623 \\
\hline-3.913 & $0.230 \pm 0.24$ & 0.031 & 1.557 & 0.009 & 4.072 & $0.210 \pm 0.59$ & 0.011 & 5.238 & 0.02 & 9.524 & $0.200 \pm 0.24$ & -0.001 & -0.5 & -0.01 & -5.00 & 0.217 \\
\hline 0.25 & $820 \pm 0.20$ & 30 & 3.797 & 22 & 2.756 & $800 \pm 1.05$ & 10 & 1.25 & -20.0 & -2.5 & $792 \pm 1.05$ & 2.00 & 0.252 & -8 & -1.010 & 801 \\
\hline-1.531 & $92.70 \pm 0.14$ & 0.56 & 0.622 & 0.05 & 0.055 & $92.75 \pm 0.24$ & 1.00 & 1.098 & 0.35 & 0.384 & $92.55 \pm 1.04$ & 1.5 & 1.638 & 0.5 & 0.546 & 92.26 \\
\hline
\end{tabular}

Note: $\mathrm{PM}=$ Pure Mysore, Improv. = improvement, $\mathrm{P} 1=$ Parental generation $1, \mathrm{CW}=$ Cocoon weight, $\mathrm{SW}=\mathrm{Shell}$ weight, Filament length, PR= Pupation rate \& $\%=$ Percentage. 
Table.3 Selection response through cellular rearing technique for the traits cocoon weight and shell weight of the silkworm Bombyx mori

\begin{tabular}{|c|c|c|c|c|c|c|c|}
\hline $\begin{array}{c}\text { Races } \\
\text { Generations }\end{array}$ & Traits & $\mathrm{C}_{108}$ & $\mathrm{NB}_{4} \mathrm{D}_{2}$ & PM & Nistari & zebra & knobbed \\
\hline $\mathbf{P}_{1}$ & \multirow{12}{*}{ 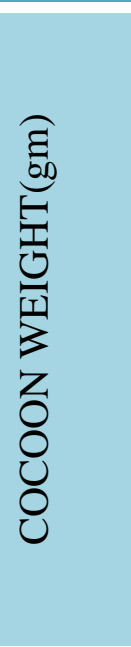 } & $1.880 \pm 0.006$ & $1.773 \pm 0.015$ & $1.033 \pm 0.009$ & $0.920 \pm 0.035$ & $1.607 \pm 0.064$ & $1.527 \pm 0.013$ \\
\hline$F_{1}$ & & $1.910 \pm 0.006$ & $1.887 \pm 0.003$ & $1.070 \pm 0.015$ & $0.933 \pm 0.028$ & $1.687 \pm 0.032$ & $1.553 \pm 0.020$ \\
\hline$F_{2}$ & & $1.920 \pm 0.012$ & $1.920 \pm 0.012$ & $1.110 \pm 0.006$ & $0.970 \pm 0.015$ & $1.723 \pm 0.034$ & $1.607 \pm 0.007$ \\
\hline$F_{3}$ & & $1.947 \pm 0.024$ & $1.987 \pm 0.003$ & $1.147 \pm 0.027$ & $1.060 \pm 0.070$ & $1.800 \pm 0.025$ & $1.623 \pm 0.028$ \\
\hline $\mathbf{F}_{4}$ & & $1.970 \pm 0.025$ & $2.027 \pm 0.013$ & $1.133 \pm 0.009$ & $1.063 \pm 0.019$ & $1.853 \pm 0.020$ & $1.693 \pm 0.030$ \\
\hline$F_{5}$ & & $1.993 \pm 0.009$ & $2.090 \pm 0.006$ & $1.153 \pm 0.003$ & $1.140 \pm 0.035$ & $1.860 \pm 0.023$ & $1.713 \pm 0.034$ \\
\hline $\mathrm{F}_{6}$ & & $2.070 \pm 0.035$ & $2.163 \pm 0.027$ & $1.253 \pm 0.003$ & $1.230 \pm 0.015$ & $1.867 \pm 0.043$ & $1.720 \pm 0.015$ \\
\hline F- Test & & $*$ & $*$ & $*$ & $*$ & $*$ & $*$ \\
\hline C.D.@ 5\% & & 0.06 & 0.042 & 0.039 & 0.11 & 0.113 & 0.07 \\
\hline $\mathrm{SE}(\mathrm{m}) \pm$ & & 0.02 & 0.014 & 0.013 & 0.036 & 0.037 & 0.023 \\
\hline $\mathrm{SE}(\mathrm{d}) \pm$ & & 0.028 & 0.019 & 0.018 & 0.051 & 0.052 & 0.033 \\
\hline C.V. (\%) & & 1.746 & 1.204 & 1.972 & 5.938 & 3.62 & 2.437 \\
\hline $\mathbf{P}_{1}$ & \multirow{12}{*}{ 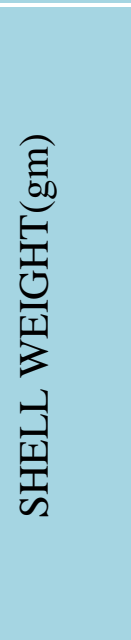 } & $0.313 \pm 0.002$ & $0.333 \pm 0.009$ & $0.127 \pm 0.001$ & $0.115 \pm 0.000$ & $0.223 \pm 0.015$ & $0.199 \pm 0.006$ \\
\hline$F_{1}$ & & $0.320 \pm 0.001$ & $0.313 \pm 0.009$ & $0.132 \pm 0.001$ & $0.126 \pm 0.001$ & $0.232 \pm 0.001$ & $0.211 \pm 0.001$ \\
\hline$F_{2}$ & & $0.326 \pm 0.003$ & $0.328 \pm 0.004$ & $0.133 \pm 0.000$ & $0.132 \pm 0.002$ & $0.238 \pm 0.001$ & $0.223 \pm 0.004$ \\
\hline$F_{3}$ & & $0.333 \pm 0.002$ & $0.330 \pm 0.006$ & $0.137 \pm 0.001$ & $0.138 \pm 0.003$ & $0.247 \pm 0.003$ & $0.240 \pm 0.001$ \\
\hline $\mathbf{F}_{4}$ & & $0.347 \pm 0.002$ & $0.337 \pm 0.004$ & $0.141 \pm 0.001$ & $0.142 \pm 0.001$ & $0.258 \pm 0.003$ & $0.249 \pm 0.001$ \\
\hline$F_{5}$ & & $0.374 \pm 0.002$ & $0.357 \pm 0.004$ & $0.147 \pm 0.001$ & $0.144 \pm 0.002$ & $0.277 \pm 0.003$ & $0.263 \pm 0.002$ \\
\hline $\mathrm{F}_{6}$ & & $0.400 \pm 0.001$ & $0.403 \pm 0.003$ & $0.152 \pm 0.001$ & $0.148 \pm 0.001$ & $0.315 \pm 0.005$ & $0.293 \pm 0.001$ \\
\hline F- Test & & $*$ & $*$ & $*$ & $*$ & $*$ & $*$ \\
\hline C.D.@ 5\% & & 0.006 & 0.018 & 0.003 & 0.005 & 0.019 & 0.009 \\
\hline $\mathrm{SE}(\mathrm{m}) \pm$ & & 0.002 & 0.006 & 0.001 & 0.002 & 0.006 & 0.003 \\
\hline $\mathrm{SE}(\mathrm{d}) \pm$ & & 0.003 & 0.008 & 0.001 & 0.002 & 0.009 & 0.004 \\
\hline C.V. (\%) & & 0.989 & 2.986 & 1.269 & 2.228 & 4.124 & 2.137 \\
\hline
\end{tabular}

Note:*=Significant $(\mathrm{P}<0.05), \mathrm{N} / \mathrm{S}=$ Non-significant $(\mathrm{P}>0.05), \mathrm{C} . \mathrm{D}=, \mathrm{SE}=$ Standard error, $\mathrm{C} . \mathrm{V}=$ 
Table.4 Selection response through cellular rearing technique for the traits filament length and pupation rate of the silkworm Bombyx mori

\begin{tabular}{|c|c|c|c|c|c|c|c|}
\hline $\begin{array}{c}\text { Races } \\
\text { Generations }\end{array}$ & Traits & $\mathrm{C}_{108}$ & $\mathrm{NB}_{4} \mathrm{D}_{2}$ & PM & Nistari & zebra & knobbed \\
\hline $\mathbf{P}_{1}$ & \multirow{12}{*}{ 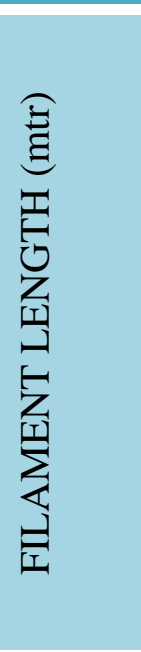 } & $899.667 \pm 0.305$ & $896.333 \pm 0.892$ & $332.667 \pm 1.454$ & $313.667 \pm 3.667$ & $807.667 \pm 3.842$ & $791.667 \pm 1.662$ \\
\hline$F_{1}$ & & $952.000 \pm 1.155$ & $895.667 \pm 2.845$ & $352.000 \pm 2.000$ & $326.000 \pm 3.215$ & $811.000 \pm 5.132$ & $799.667 \pm 0.308$ \\
\hline$F_{2}$ & & $962.667 \pm 1.446$ & $920.333 \pm 0.360$ & $363.667 \pm 1.335$ & $337.333 \pm 1.855$ & $822.000 \pm 4.726$ & $813.333 \pm 3.530$ \\
\hline$F_{3}$ & & $968.667 \pm 1.851$ & $952.333 \pm 1.460$ & $371.000 \pm 0.577$ & $351.333 \pm 6.333$ & $830.333 \pm 4.335$ & $824.000 \pm 3.512$ \\
\hline $\mathbf{F}_{4}$ & & $999.667 \pm 0.301$ & $995.667 \pm 0.301$ & $380.000 \pm 0.577$ & $351.333 \pm 6.333$ & $841.667 \pm 4.053$ & $830.333 \pm 5.813$ \\
\hline$F_{5}$ & & $1106.000 \pm 1.000$ & $1192.000 \pm 1.528$ & $385.000 \pm 1.732$ & $371.667 \pm 4.410$ & $864.000 \pm 4.163$ & $839.667 \pm 4.175$ \\
\hline $\mathrm{F}_{6}$ & & $1201.333 \pm 1.843$ & $1200.333 \pm 0.250$ & $408.333 \pm 1.200$ & $377.667 \pm 4.334$ & $870.000 \pm 9.504$ & $854.333 \pm 3.846$ \\
\hline F- Test & & $*$ & $*$ & $*$ & $*$ & $*$ & $*$ \\
\hline C.D.@ 5\% & & 3.935 & 4.279 & 4.174 & 13.971 & 16.627 & 11.203 \\
\hline $\mathrm{SE}(\mathrm{m}) \pm$ & & 1.285 & 1.397 & 1.363 & 4.562 & 5.429 & 3.658 \\
\hline $\mathrm{SE}(d) \pm$ & & 1.817 & 1.976 & 1.927 & 6.451 & 7.678 & 5.173 \\
\hline C.V. (\%) & & 0.22 & 0.24 & 0.637 & 2.277 & 1.126 & 0.771 \\
\hline $\mathbf{P}_{1}$ & \multirow{12}{*}{ 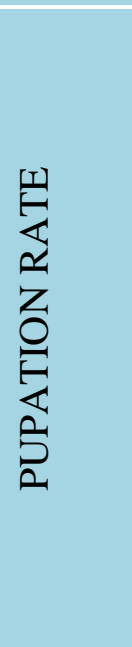 } & $93.150 \pm 0.042$ & $94.460 \pm 0.034$ & $93.080 \pm 0.030$ & $91.573 \pm 0.037$ & $90.077 \pm 0.042$ & $91.073 \pm 0.019$ \\
\hline$F_{1}$ & & $92.993 \pm 0.058$ & $94.220 \pm 0.027$ & $93.537 \pm 0.042$ & $91.820 \pm 0.000$ & $89.687 \pm 0.212$ & $90.403 \pm 0.297$ \\
\hline$F_{2}$ & & $92.120 \pm 0.024$ & $94.020 \pm 0.027$ & $93.890 \pm 0.000$ & $92.503 \pm 0.028$ & $88.787 \pm 0.211$ & $89.270 \pm 0.120$ \\
\hline$F_{3}$ & & $92.503 \pm 0.047$ & $93.677 \pm 0.015$ & $94.637 \pm 0.032$ & $92.903 \pm 0.000$ & $87.627 \pm 0.309$ & $88.680 \pm 0.293$ \\
\hline $\mathbf{F}_{4}$ & & $92.637 \pm 0.144$ & $93.463 \pm 0.000$ & $94.953 \pm 0.022$ & $93.400 \pm 0.024$ & $87.437 \pm 0.337$ & $87.030 \pm 0.299$ \\
\hline$F_{5}$ & & $91.553 \pm 0.019$ & $93.283 \pm 0.023$ & $95.863 \pm 0.057$ & $93.890 \pm 0.000$ & $86.683 \pm 0.159$ & $86.487 \pm 0.024$ \\
\hline $\mathrm{F}_{6}$ & & $91.117 \pm 0.081$ & $93.107 \pm 0.007$ & $95.987 \pm 0.016$ & $94.420 \pm 0.081$ & $86.450 \pm 0.400$ & $86.517 \pm 0.517$ \\
\hline F- Test & & $*$ & $*$ & $*$ & $*$ & $*$ & $*$ \\
\hline C.D.@ 5\% & & 0.218 & 0.058 & 0.093 & 0.103 & 0.808 & 0.855 \\
\hline $\mathrm{SE}(\mathrm{m}) \pm$ & & 0.071 & 0.019 & 0.03 & 0.034 & 0.264 & 0.279 \\
\hline $\mathrm{SE}(\mathrm{d}) \pm$ & & 0.1 & 0.027 & 0.043 & 0.047 & 0.373 & 0.395 \\
\hline C.V. $(\%)$ & & 0.133 & 0.035 & 0.056 & 0.063 & 0.518 & 0.546 \\
\hline
\end{tabular}

Note: $*=$ Significant $(\mathrm{P}<0.05), \mathrm{N} / \mathrm{S}=$ Non-significant $(\mathrm{P}>0.05), \mathrm{C} . \mathrm{D}=$ Critical difference, $\mathrm{SE}=$ Standard error, $\mathrm{C} . \mathrm{V}=\mathrm{Coefficient}$ of variation 
Table.5 Selection response through mass rearing technique for the traits cocoon weight and shell weight of the silkworm Bombyx mori

\begin{tabular}{|c|c|c|c|c|c|c|c|}
\hline $\begin{array}{c}\text { Races } \\
\text { Generations }\end{array}$ & Traits & $\mathrm{C}_{108}$ & $\mathrm{NB}_{4} \mathrm{D}_{2}$ & PM & Nistari & zebra & knobbed \\
\hline $\mathbf{P}_{1}$ & \multirow{12}{*}{ 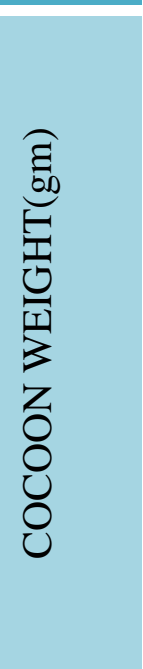 } & $1.863 \pm 0.022$ & $1.757 \pm 0.029$ & $1.047 \pm 0.033$ & $0.880 \pm 0.006$ & $1.560 \pm 0.023$ & $1.543 \pm 0.070$ \\
\hline$F_{1}$ & & $1.960 \pm 0.010$ & $1.940 \pm 0.025$ & $1.087 \pm 0.041$ & $0.987 \pm 0.003$ & $1.630 \pm 0.110$ & $1.640 \pm 0.057$ \\
\hline$F_{2}$ & & $1.983 \pm 0.009$ & $1.880 \pm 0.006$ & $1.167 \pm 0.037$ & $1.053 \pm 0.032$ & $1.640 \pm 0.067$ & $1.717 \pm 0.037$ \\
\hline$F_{3}$ & & $1.943 \pm 0.084$ & $1.947 \pm 0.003$ & $1.093 \pm 0.009$ & $0.977 \pm 0.015$ & $1.733 \pm 0.023$ & $1.607 \pm 0.043$ \\
\hline $\mathbf{F}_{4}$ & & $2.047 \pm 0.052$ & $2.053 \pm 0.032$ & $1.167 \pm 0.022$ & $1.120 \pm 0.015$ & $1.763 \pm 0.065$ & $1.670 \pm 0.036$ \\
\hline$F_{5}$ & & $1.997 \pm 0.073$ & $1.927 \pm 0.037$ & $1.093 \pm 0.052$ & $0.973 \pm 0.019$ & $1.710 \pm 0.072$ & $1.600 \pm 0.087$ \\
\hline$F_{6}$ & & $1.880 \pm 0.060$ & $1.847 \pm 0.073$ & $1.113 \pm 0.015$ & $0.880 \pm 0.100$ & $1.730 \pm 0.117$ & $1.550 \pm 0.085$ \\
\hline F- Test & & $\mathrm{N} / \mathrm{S}$ & $*$ & $\mathrm{~N} / \mathrm{S}$ & $*$ & $\mathrm{~N} / \mathrm{S}$ & $\mathrm{N} / \mathrm{S}$ \\
\hline C.D.@ $9 \%$ & & $\mathrm{~N} / \mathrm{S}$ & 0.111 & $\mathrm{~N} / \mathrm{S}$ & 0.126 & $\mathrm{~N} / \mathrm{S}$ & $\mathrm{N} / \mathrm{S}$ \\
\hline $\mathrm{SE}(\mathrm{m}) \pm$ & & 0.053 & 0.036 & 0.033 & 0.041 & 0.076 & 0.062 \\
\hline $\mathrm{SE}(\mathrm{d}) \pm$ & & 0.074 & 0.051 & 0.047 & 0.058 & 0.108 & 0.088 \\
\hline C.V. $(\%)$ & & 4.663 & 3.302 & 5.148 & 7.135 & 7.856 & 6.685 \\
\hline $\mathbf{P}_{1}$ & \multirow{12}{*}{ 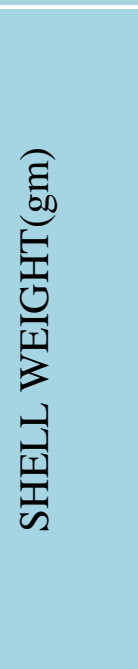 } & $0.316 \pm 0.003$ & $0.331 \pm 0.005$ & $0.124 \pm 0.002$ & $0.114 \pm 0.000$ & $0.235 \pm 0.009$ & $0.199 \pm 0.000$ \\
\hline $\mathbf{F}_{1}$ & & $0.337 \pm 0.002$ & $0.326 \pm 0.003$ & $0.139 \pm 0.001$ & $0.128 \pm 0.001$ & $0.296 \pm 0.003$ & $0.211 \pm 0.000$ \\
\hline $\mathbf{F}_{2}$ & & $0.340 \pm 0.008$ & $0.322 \pm 0.001$ & $0.142 \pm 0.004$ & $0.136 \pm 0.003$ & $0.307 \pm 0.009$ & $0.229 \pm 0.003$ \\
\hline $\mathbf{F}_{3}$ & & $0.345 \pm 0.011$ & $0.333 \pm 0.006$ & $0.134 \pm 0.003$ & $0.126 \pm 0.003$ & $0.299 \pm 0.000$ & $0.229 \pm 0.006$ \\
\hline $\mathbf{F}_{4}$ & & $0.359 \pm 0.016$ & $0.392 \pm 0.004$ & $0.145 \pm 0.003$ & $0.135 \pm 0.002$ & $0.312 \pm 0.006$ & $0.233 \pm 0.006$ \\
\hline$F_{5}$ & & $0.359 \pm 0.016$ & $0.389 \pm 0.010$ & $0.138 \pm 0.007$ & $0.121 \pm 0.004$ & $0.300 \pm 0.006$ & $0.215 \pm 0.010$ \\
\hline $\mathrm{F}_{6}$ & & $0.350 \pm 0.005$ & $0.376 \pm 0.018$ & $0.135 \pm 0.005$ & $0.118 \pm 0.001$ & $0.285 \pm 0.004$ & $0.213 \pm 0.008$ \\
\hline F- Test & & $\mathrm{N} / \mathrm{S}$ & $*$ & $\mathrm{~N} / \mathrm{S}$ & $*$ & $*$ & $*$ \\
\hline C.D.@ 5\% & & $\mathrm{N} / \mathrm{S}$ & 0.026 & $\mathrm{~N} / \mathrm{S}$ & 0.007 & 0.019 & 0.019 \\
\hline $\mathrm{SE}(\mathrm{m}) \pm$ & & 0.01 & 0.008 & 0.004 & 0.002 & 0.006 & 0.006 \\
\hline $\mathrm{SE}(d) \pm$ & & 0.014 & 0.012 & 0.006 & 0.003 & 0.009 & 0.009 \\
\hline C.V. (\%) & & 5.112 & 4.138 & 5.168 & 3.308 & 3.648 & 4.791 \\
\hline
\end{tabular}

Note: $*=$ Significant $(\mathrm{P}<0.05), \mathrm{N} / \mathrm{S}=$ Non-significant $(\mathrm{P}>0.05), \mathrm{C} . \mathrm{D}=$ Critical difference, $\mathrm{SE}=$ Standard error, $\mathrm{C} . \mathrm{V}=$ Coefficient of variation 
Table.6 Selection response through mass rearing technique for the traits filament length and pupation rate of the silkworm Bombyx mori

\begin{tabular}{|c|c|c|c|c|c|c|c|}
\hline Races & Traits & $\mathrm{C}_{108}$ & $\mathrm{NB}_{4} \mathrm{D}_{2}$ & PM & Nistari & zebra & knobbed \\
\hline $\mathbf{P}_{1}$ & \multirow{12}{*}{ 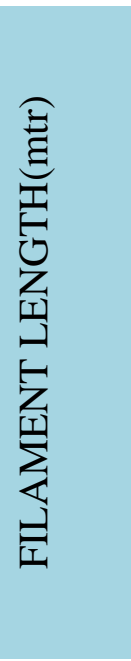 } & $897.00 \pm 3.00$ & $897.67 \pm 1.45$ & $330.67 \pm 0.34$ & $314.00 \pm 3.51$ & $804.67 \pm 4.67$ & $792.33 \pm 3.39$ \\
\hline$F_{1}$ & & $996.67 \pm 3.84$ & $975.33 \pm 8.97$ & $358.67 \pm 4.10$ & $339.00 \pm 5.51$ & $854.00 \pm 4.93$ & $803.00 \pm 4.00$ \\
\hline $\mathbf{F}_{2}$ & & $997.67 \pm 1.45$ & $992.67 \pm 3.71$ & $368.00 \pm 1.73$ & $365.00 \pm 2.31$ & $859.00 \pm 2.65$ & $823.00 \pm 34.01$ \\
\hline $\mathbf{F}_{3}$ & & $1033.67 \pm 33.17$ & $999.67 \pm 6.36$ & $365.33 \pm 4.84$ & $343.67 \pm 2.67$ & $864.00 \pm 5.57$ & $799.00 \pm 0.58$ \\
\hline $\mathbf{F}_{4}$ & & $1081.00 \pm 42.19$ & $1103.67 \pm 60.94$ & $396.33 \pm 1.33$ & $375.00 \pm 2.31$ & $868.67 \pm 5.24$ & $817.33 \pm 3.18$ \\
\hline$F_{5}$ & & $999.00 \pm 0.58$ & $1066.33 \pm 66.84$ & $391.33 \pm 4.48$ & $366.00 \pm 2.65$ & $863.33 \pm 6.01$ & $807.67 \pm 8.17$ \\
\hline $\mathrm{F}_{6}$ & & $990.33 \pm 4.49$ & $984.00 \pm 17.01$ & $373.33 \pm 4.37$ & $358.67 \pm 4.81$ & $832.33 \pm 1.86$ & $793.33 \pm 2.97$ \\
\hline F- Test & & $*$ & $*$ & $*$ & $*$ & $*$ & $\mathrm{~N} / \mathrm{S}$ \\
\hline C.D.@ $9 \%$ & & 62.623 & 107.39 & 10.63 & 11.015 & 14.229 & $\mathrm{~N} / \mathrm{S}$ \\
\hline $\mathrm{SE}(\mathrm{m}) \pm$ & & 20.448 & 35.065 & 3.471 & 3.597 & 4.646 & 13.468 \\
\hline $\mathrm{SE}(d) \pm$ & & 28.918 & 49.59 & 4.909 & 5.087 & 6.571 & 19.047 \\
\hline C.V. (\%) & & 3.544 & 6.057 & 1.629 & 1.772 & 0.947 & 2.898 \\
\hline $\mathbf{P}_{1}$ & \multirow{12}{*}{ 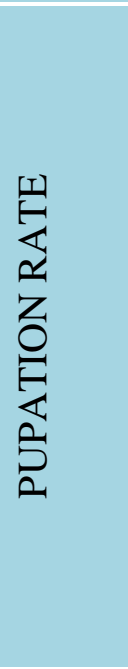 } & $93.140 \pm 0.023$ & $94.397 \pm 0.009$ & $93.087 \pm 0.018$ & $91.330 \pm 0.088$ & $90.187 \pm 0.182$ & $90.067 \pm 0.004$ \\
\hline $\mathbf{F}_{1}$ & & $94.237 \pm 0.142$ & $94.490 \pm 0.025$ & $93.543 \pm 0.003$ & $92.080 \pm 0.083$ & $90.553 \pm 0.059$ & $91.527 \pm 0.041$ \\
\hline $\mathbf{F}_{2}$ & & $94.907 \pm 0.036$ & $94.600 \pm 0.052$ & $94.217 \pm 0.007$ & $92.597 \pm 0.653$ & $91.583 \pm 0.048$ & $92.097 \pm 0.060$ \\
\hline $\mathrm{F}_{3}$ & & $93.837 \pm 0.051$ & $93.860 \pm 0.014$ & $93.463 \pm 0.105$ & $91.243 \pm 0.043$ & $89.577 \pm 0.221$ & $90.337 \pm 0.161$ \\
\hline $\mathbf{F}_{4}$ & & $94.153 \pm 0.000$ & $94.577 \pm 0.034$ & $94.110 \pm 0.058$ & $93.663 \pm 0.037$ & $90.553 \pm 0.081$ & $90.533 \pm 0.142$ \\
\hline$F_{5}$ & & $95.253 \pm 0.026$ & $95.257 \pm 0.202$ & $94.443 \pm 0.104$ & $93.590 \pm 0.042$ & $90.620 \pm 0.064$ & $91.103 \pm 0.069$ \\
\hline$F_{6}$ & & $96.567 \pm 0.000$ & $95.953 \pm 0.037$ & $95.187 \pm 0.135$ & $94.127 \pm 0.047$ & $91.697 \pm 0.099$ & $91.540 \pm 0.010$ \\
\hline F- Test & & $*$ & $*$ & $*$ & $*$ & $*$ & $*$ \\
\hline C.D. @ 5\% & & 0.186 & 0.252 & 0.244 & 0.774 & 0.382 & 0.276 \\
\hline $\mathrm{SE}(\mathrm{m}) \pm$ & & 0.061 & 0.082 & 0.08 & 0.253 & 0.125 & 0.09 \\
\hline $\mathrm{SE}(d) \pm$ & & 0.086 & 0.117 & 0.113 & 0.358 & 0.176 & 0.128 \\
\hline C.V. $(\%)$ & & 0.111 & 0.151 & 0.147 & 0.473 & 0.238 & 0.172 \\
\hline
\end{tabular}

Note: $*=$ Significant $(\mathrm{P}<0.05), \mathrm{N} / \mathrm{S}=$ Non-significant $(\mathrm{P}>0.05), \mathrm{C} . \mathrm{D}=$ Critical difference, $\mathrm{SE}=$ Standard error, $\mathrm{C} . \mathrm{V}=$ Coefficient of variation 
Fig.1\&2 Showing variation in single cocoon weight \& single shell weight through selection response of cellular rearing technique
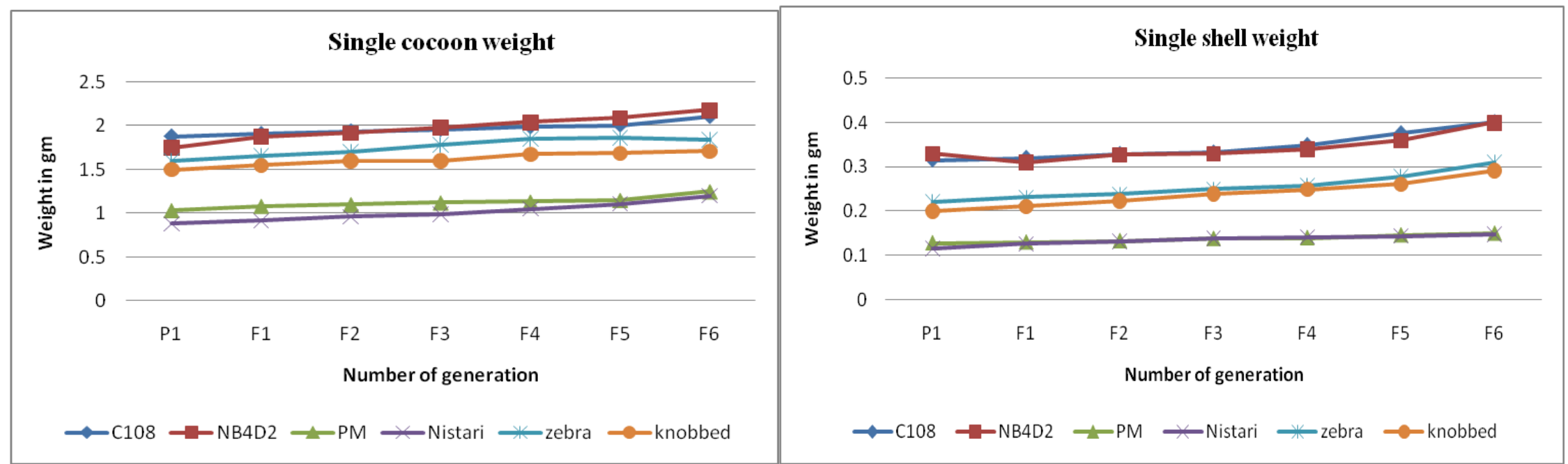

Fig.3\&4 Showing variation in filament length \& pupation rate through selection response of cellular rearing technique
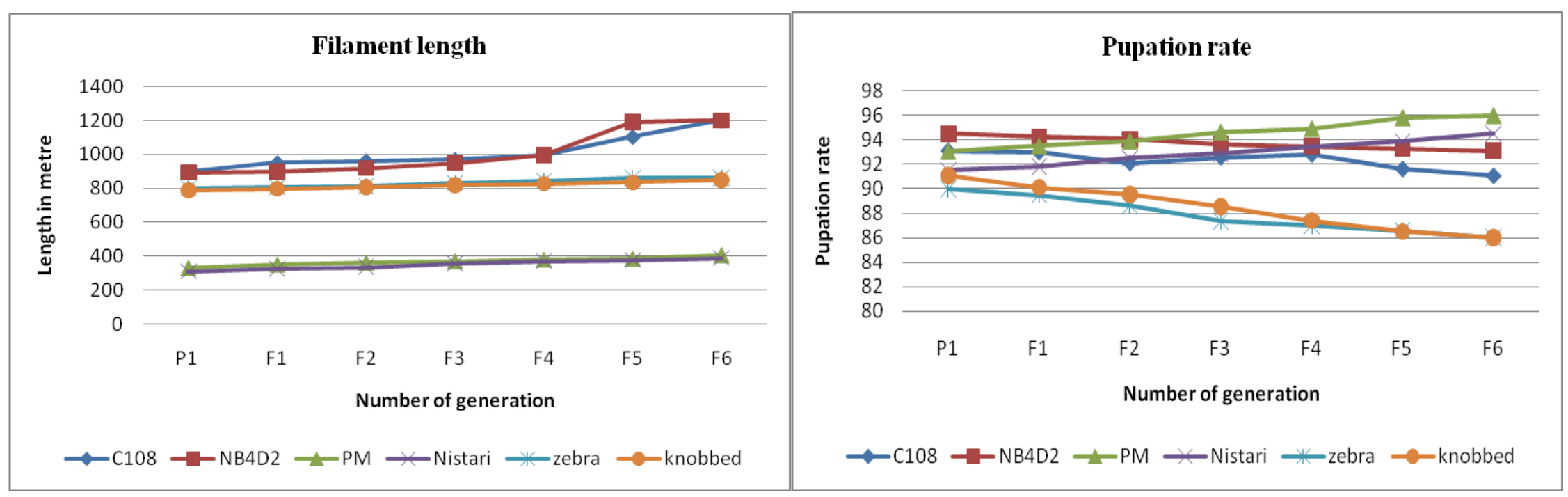
Fig.5\&6 Showing variation in single cocoon weight \& single shell weight through selection response of mass rearing technique
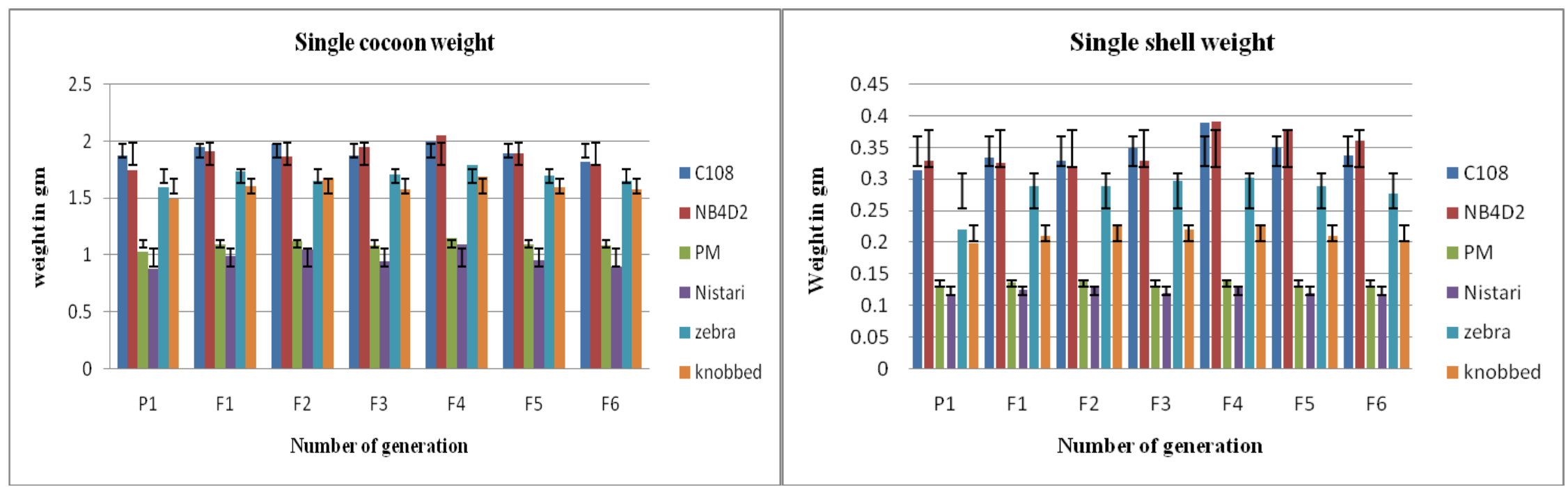

Fig.7\&8 Showing variation in filament length \& pupation rate through selection response of mass rearing technique

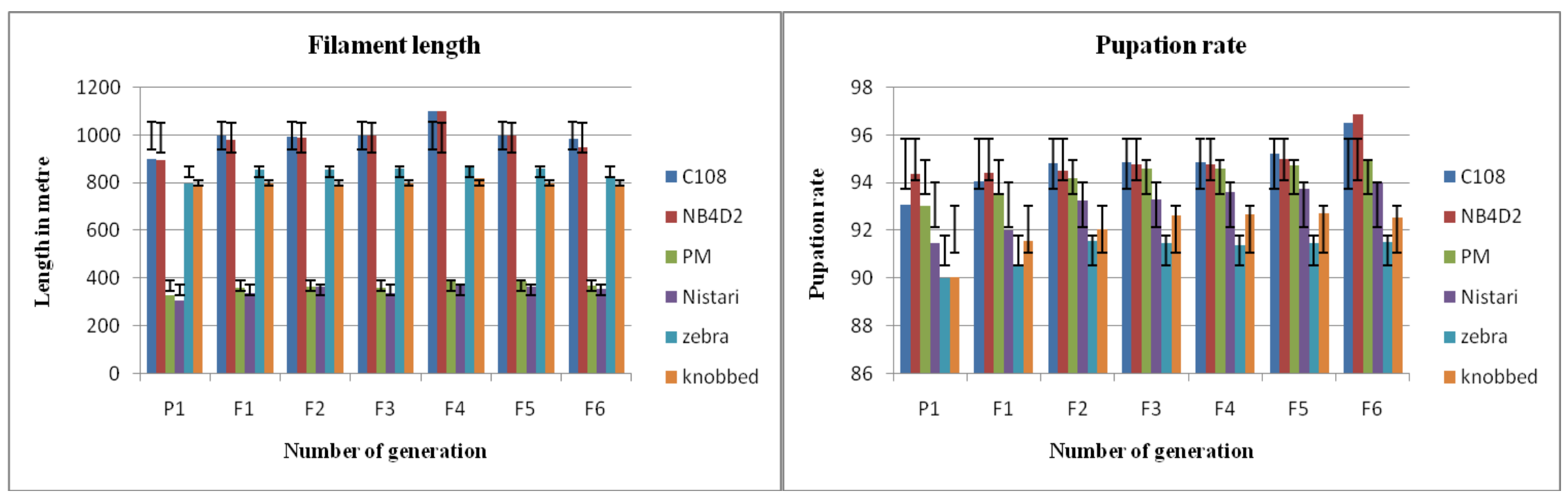


Fig.9 Merit ratio for different tarits between $\mathrm{F}_{6}$ and $\mathrm{P}$ generations through cellular rearing technique in the silkworm Bombyx mori

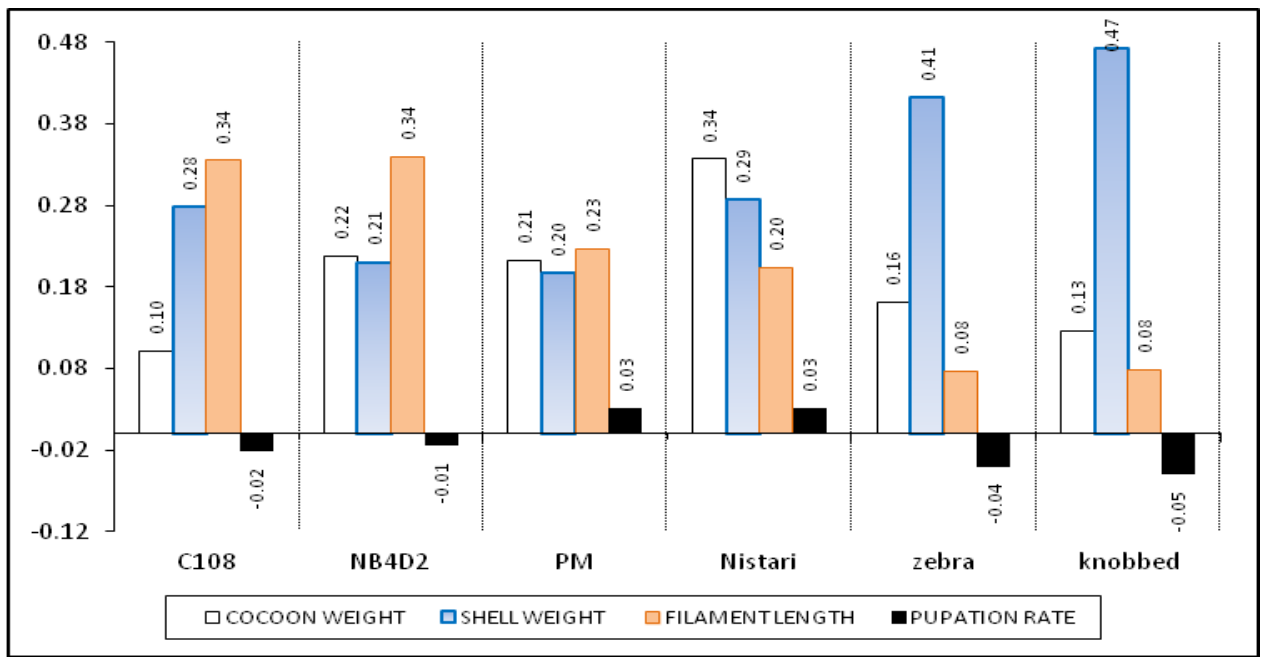

Fig.10 Merit ratio for different tarits between $\mathrm{F}_{4}$ and $\mathrm{F}_{6}$ generations through mass rearing technique in the silkworm Bombyx mori

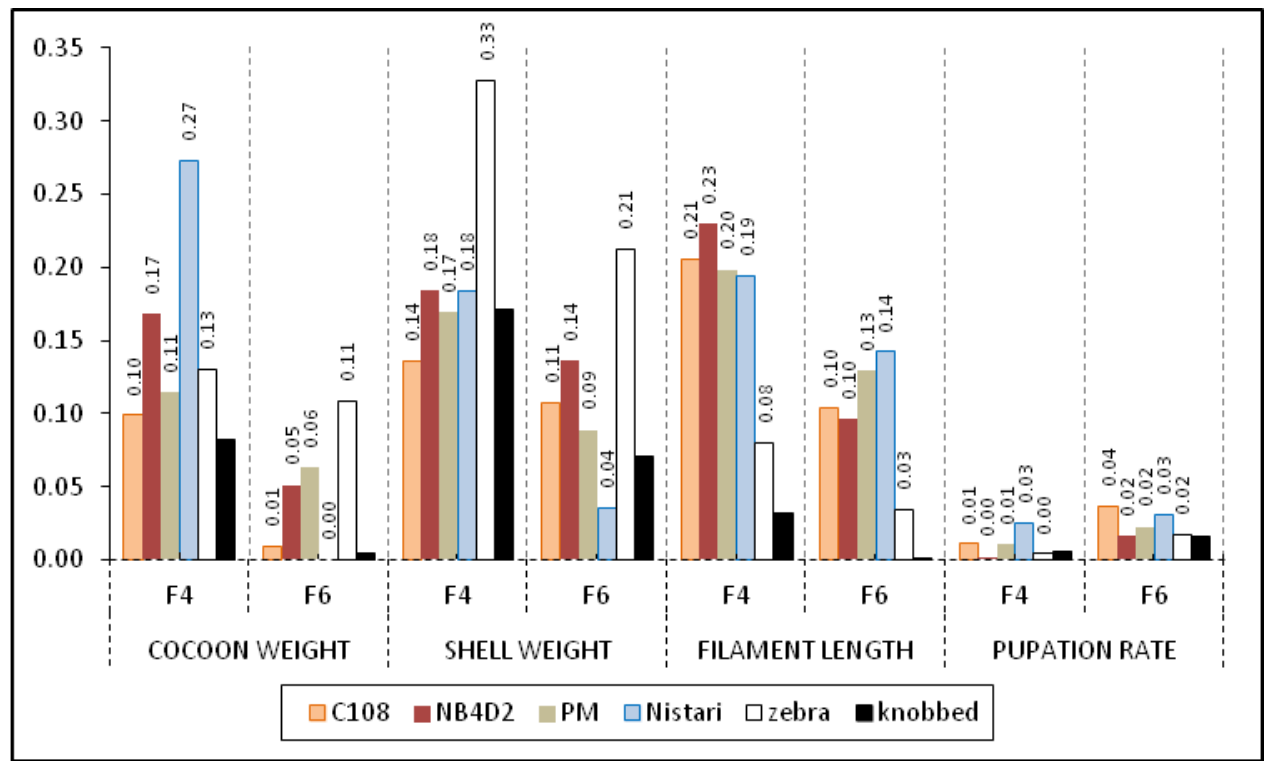

However, this kind of phenomenon was also observed for the traits like shell weight (Fig.5 and 6) \& filament length (Fig. 7) at from F1 to F6 generations in $\mathrm{NB}_{4} \mathrm{D}_{2}, \mathrm{PM}$, Nistari, zebra \& knobbed races/mutants. But, this kind of phenomenon (heterosis \& inbreeding depression) was not evident for the trait like pupation rate (Fig. 8) instead there was increasing (heterosis) significantly $(\mathrm{P}<0.05)$ in pupation rate across the six generations in all the selected races/mutants of the silkworm Bombyx mori. The recorded triplicate data was subjected to opistat analysis tool and obtained results in relation to selection response through cellular rearing technique for the traits cocoon weight, shell weight of the silkworm Bombyx mori is depicted in table 3. A highest of $2.07 \mathrm{~g}$ was revealed 
during F6 generation compare to the rest of generations in $\mathrm{C} 108$ race and remaining races were recorded variable in expression of quantitative traits. The C.D @ 5\% value was higher of 0.113 in zebra mutant followed by Nistari (0.11), knobbed (0.07), C108 (0.06), $\mathrm{NB}_{4} \mathrm{D}_{2}(0.042) \& \mathrm{PM}(0.039)$ across the six generations. The percent of C.V (\%) was higher in case of Nistari (5.938) \& minimum of 1.204 was recorded in $\mathrm{NB}_{4} \mathrm{D}_{2}$ race. Further, same table depicted the shell weight, which is highest expressed in F6 generation for $\mathrm{C}_{108}$ $(0.100 \mathrm{~g})$ among the six generations \& C.D @ $5 \%$ was seen in zebra mutant $(0.019) \&$ remaining races shown variable in percent. Moreover, both the traits (Cocoon weight \& shell weight) revealed significant $(\mathrm{P}<0.05)$ for the selected races/mutants across the six generations.

Further, the data in regard to selection response through cellular rearing method for the traits filament length and pupation rate is presented in Table 4. The data for the trait filament length clearly shown the C.D @ 5\% value higher of 16.627 in nistari race and remaining races/mutants recorded intermediary values. As such for the trait pupation rate was highest of 0.855 C.D @ $5 \%$ in knobbed mutant \& remaining five races/mutants were recorded intermediary values. It is very interesting that, both the traits were shown statistically significant $(\mathrm{P}<0.05)$. Moreover, estimates of selection response through mass rearing method for the traits cocoon weight \& shell weight was depicted in Table 5. It was clearly shown cocoon weight higher $(2.047 \mathrm{~g})$ during F4 generation in $\mathrm{C} 108$ race \& $2.053 \mathrm{~g}$ maximum was recorded in $\mathrm{NB}_{4} \mathrm{D}_{2}$ race during same generation. The data for the trait shell weight higher of $0.359 \mathrm{~g}$ was revealed during F4 \& F5 generations in $\mathrm{C}_{108}$ alone $\& \mathrm{NB}_{4} \mathrm{D}_{2}$ recorded higher shell weight than $\mathrm{C} 108$ during said generations. It is very important to note, the race/mutants $\left(\mathrm{C}_{108}, \mathrm{PM}\right.$, zebra \& knobbed $)$ were shown statistically non-significant (P>0.05) except $\mathrm{NB}_{4} \mathrm{D}_{2} \&$ nistari, which were expressed statistically significant $(\mathrm{P}<0.05)$. At the same time, on the other hand for the trait shell weight all the selected races/mutants recorded statistically significant except $\mathrm{C}_{108}$ \& PM were evident with statistically nonsignificant $(\mathrm{P}>0.05)$. Furthermore, Table 6 represented the data with regard to selection response through mass rearing for the trait filament length \& pupation rate of the silkworm Bombyx mori. All the races/mutants were shown statistically significant $(\mathrm{P}<0.05)$ for the both traits (filament length $\&$ pupation rate) except knobbed mutant, which was revealed statistically expressed nonsignificant $(\mathrm{P}>0.05)$. Further, Merit ratio for different traits between $\mathrm{F}_{6}$ and $\mathrm{P}$ generations through cellular rearing technique in the silkworm Bombyx mori were clear distingushed between the generations and traits selection response (Fig. 9), the ratio for the trait cocoon weight in Nistari, NB4D2 and PM maybe due to Pupation rate, for the trait shell weight in Knobbed and Zebra because of Pupation rate, for the filament length in $\mathrm{C} 108$ and NB4D2 probably because of silk gland weight and pupation rate showed higher range for response selection in Nistari and PM. Moreover, merit ratio for different tarits between $\mathrm{F}_{4}, \mathrm{~F}_{6}$ and $\mathrm{P}$ generations through mass rearing technique in the silkworm Bombyx mori was depicted in Figure 10 showed better expression of the traits for selection response during F4 generation than F6 generation wherein deterioration of traits noticed through mass rearing technique. Several investigators have researched on selection response in silkworm are Hajian et al., (2011).

However, studies of selection response shown variable in results for both the methods, because one of the important method of breeding for silkworm race/strains with polygentically inherited characters is selection 
for desired characters. There were several investigators, who have worked by utilizing batch/mass as well as individual selection methods to determine even the effect of different disease of the silkworm during many generations for evolutionary studies. Among them, Funada (1968) have observed silkworm resistant to IFV infection by selection method through repeated exposure of the virus \& they succeeded finally resistant strains after several generations.

Further, Watanabe, (1967) have exposed for CPV upto 8 generations, but there was not resistant till 4 generation but finally got resistant strains during 5 generation through proper selection procedure. Ultimately, Since, in the present work under the six generations of the selection response utilizing cellular and mass systems revealed considerable increase in selected traits like, cocoon weight, shell weight \& filament length, which is well revealed by Falconer and Mackay, (1996) except pupation rate through cellular rearing method.

But, this kind of phenomenon was not observed through mass selection, all the selected traits variable in expression of cocoon weight, shell weight $\&$ filament length except pupation rate, which was shown increased considerably across the six generations. Thereby, it is concluded with the response of two selection methods was differed in the expression of the cocoon weight, shell weight and filament across the six generations. On other hand, pupation rate is also responded constant direction expression by cellular as well as mass methods of selection. Hence, cellular as well as mass methods of selection were played an important role, which are constantly helpful for the expression/improvement of desired quantitative traits of the silkworm Bombyx mori.

Acknowledgements
The authors wish to express sincere thanks to University Grants Commission for providing the funds. We wish to thank the Chairman, Department of Studies in Sericulture Science, Manasagangotri, University of Mysore, Mysuru for extending the laboratory facilities to carry out the research work.

\section{References}

Bijma, P. (2006) Estimating maternal genetic effects in livestock, J.Anim.SCI., 84: 800-806.

Bijma, P., Muir, W.M and Van Arendonk, J.A. (2007a). Multilevel selection 1: Quantitative genetics of inheritance and response to selection. Genetics, 175: 277-288.

Bohren, B. B., Kinney, T., Wilson, S. P and Lowe, P. C. (1970) Genetic gains in annual egg production from selection on part-record percent production in the fowl, Genetics 65: 55-667.

Chen, C.Y., Johnson,R.K., Newman, S and Van Vleck L.D. (2007) A general revoew of competition genetic effects with an emphasis on swine breeding, Genetics and molecular research, 6(3): 594-606.

Dobzhansky, T. (1950) Genetics of natural populations. XIX. Origin of heterosis through natural selection in populations Drosophila pseudobscura, Genetics, 35 288-302.

Ehsan Hajian, Alireza Seidavi and Abolghasem Larvvaf. (2011) Estimation of response to selection in three silkworm commercial pure lines with oval cocoon, Annals of Biological Research, 2(1): 215-225.

Falconer, D.S and Mackay, T.F.C. (1996) Introduction to quantitative genetics, $4^{\text {th }}$ edn. Longman, New York.

Funada, T. (1968) Ibid, 37: 281-287. 
Hajian, E., Seidavi, A and Lavvaf, A. (2011) Estimation of response to selection in three silkworm commercial pure lines with oval cocoons. Annals of Biological Re- search, 2, 215-225.

He, Y and Oshiki, T. (1984) Study on cross breeding of a robust silkworm race for summer and autumn rearing at low latitude area in China, J.Seric.Sci.Jpn., 53:320-324.

Kinney, T.B. Jr ${ }^{\circ}$, B.Bo Bohren, J.V. Craig, and P.C. Lowe, (1970) Responses to individual, family or index selection for short term rate of egg production in chickens. Poul. Sci. 49:1052-1064.

Krishnaswami, S (1978) New technology of silkworm rearing, Bulletin No. 2, Central Sericultural Research and Training Institute, Mysore, India, 23.

Lush, J.L., (1947) Family merit and individual merit as bases for selection. Amer. Nat. 81: 241-261, 362-379.

Nirmal Kumar, S. (1995) Studies on the synthesis of appropriate silkworm breed (Bombyx mori L) for tropics, Ph.D. Thesis, University of Mysore, Mysore, 316.

Robertson, A., (1960) A theory of limits in artificial selection. Proc. RoyalSoc. 153, Vol. B., 234-249.
Singh, T., Sekharaiah, C and Samson, M. V. (1998) Correlation and heritability analysis in the silkworm, Bombyx mori L., Sericologia, 38, 1-13.

Sudhakar Rao, P. (2003) Studies on the evoluation of adaptive bivoltine breeds of silkworm, Bombyx mori $\mathrm{L}$ for tropical climates, Ph.D Thesis, University of Mysore, Mysore.

Suresh Kumar, N., Saha, A.K and Bindroo, B.B. (2013) Selection of breeding resource materials of Bombyx mori L.for the development of bivoltine hybrids suitable for West Bengal, Universal Journal of Environmental Research and Technology, 3(1), 28-38.

Van Vleck L.D., Cundif, L.V and Koch, R.M. (2007) Effect of competition on gain in feedlot bulls from Hereford selection lines, J.Anim.Sci. 85: 1625-1633.

Watanabe, H. (1967) J. Invertebr. Pathol, 9: 474-479.

Wilson, S.P., P.V. Blair, W.H. Kyle and A.E. Bell, (1968) The influence of selection and mating systems on larval weight in Tribolium. J. Heredity 59: 313-317.

Yokoyama, T (1963) Sericulture, A. Rv. Ent., 8: 287-306.

\section{How to cite this article:}

Shivkumar, M.N. Ramya, E. Talebi and Subramanya, G. 2018. Selection Response for the Expression of Quantitative Traits in the Mulberry Silkworm Bombyx mori L. Int.J.Curr.Microbiol.App.Sci. 7(11): 3456-3474. doi: https://doi.org/10.20546/ijcmas.2018.711.396 